Penultimate version; to be published in Visual Cognition.

\title{
THE EFFECT OF AGE-OF-ACQUISITION:
}

\author{
PARTLY FREQUENCY-RELATED
}

\author{
PARTLY FREQUENCY-INDEPENDENT
}

\section{Marc Brysbaert $^{1} \quad$ Mandy Ghyselinck $^{2}$}

\author{
${ }^{1}$ Royal Holloway, University of London, UK \\ ${ }^{2}$ Ghent University, Belgium
}

\author{
Address : $\quad$ Marc Brysbaert \\ Department of Psychology \\ Royal Holloway \\ University of London \\ Egham \\ Surrey TW20 0EX \\ United Kingdom \\ Tel. $\quad 44-1784-443524$ \\ Fax. $\quad 44-1784-434347$ \\ e-mail: Marc.Brysbaert@rhul.ac.uk
}




\begin{abstract}
A review of multitask investigations on the locus of the age-of-acquisition (AoA) effect in the English, Dutch, and French language reveals two main findings. First, for most tasks there is near perfect correlation between the magnitude of the AoA effect and the magnitude of the frequency effect, even though the stimuli were selected so that both variables were orthogonal. This frequency-related AoA effect is as large as the frequency effect, despite the fact that the range of AoA values is more restricted than the range of frequency values. Second, a frequencyindependent AoA effect is observed in object naming and word associate generation. Different explanations of the frequency-related and the frequency-independent AoA effects are reviewed and evaluated.
\end{abstract}




\section{THE EFFECT OF AGE-OF-ACQUISITION: \\ PARTLY FREQUENCY-RELATED \\ PARTLY FREQUENCY-INDEPENDENT}

When the issue of age-of-acquisition (AoA) in visual word recognition was revitalised in the mid 1990s (Morrison \& Ellis, 1995; Brysbaert, 1996; Gerhand \& Barry, 1998), hypotheses were dominated by the idea that the effect originated from the verbal output system. The main reason for this was that whereas the AoA effect had been found in each and every naming experiment, it had failed to emerge in a perceptual identification task with tachistoscopically presented words (Gilhooly \& Logie, 1981) and in a semantic classification task where participants had to indicate whether pictures referred to naturally-occurring things or to manmade objects (Morrison, Ellis, \& Quinlan, 1992).

\section{Multitask investigations of the locus of the AoA-effect}

To examine the locus of the AoA-effect, a number of authors have designed multitask investigations. Gerhand and Barry (1998, 1999a, 1999b) were the first to launch the idea that if the AoA effect was due to the activation of phonology in the verbal output system, then it should be reduced in a task that discourages such activation. So, the AoA-effect should be minimal (or even absent) in a lexical decision task with pseudohomophones (such as brane, maik) as nonword targets. In contrast, it could be larger in a speeded naming task, where participants were encouraged to read aloud words as fast as possible (and the task capitalised maximally on the spoken output). The same set of 64 words was used, consisting of 16 early-acquired ( $<6$ years old ) high-frequency (> 50/million) words, 16 early-acquired low-frequency ( $<$ 5/million) words, 16 late-acquired (> 9 years old) high-frequency words, and 16 late-acquired low-frequency words. Table 1 shows Gerhand and Barry’s results for six different tasks: naming without time 
pressure (immediate naming), naming after the stimulus word has been seen for more than a second (delayed naming), naming under time pressure (speeded naming), lexical decision with legal nonwords (such as brank), lexical decision with illegal nonwords (such as brnkf), and lexical decision with pseudohomophones (such as brane). Whereas Gerhand and Barry focussed on the (small) differences in the magnitudes of the AoA and frequency effects as a function of the task, we were particularly struck by the strong correlation of the AoA and frequency effects across tasks $(r=.96, n=6)$. In addition, a linear regression analysis indicated that the intercept did not differ significantly from 0 , suggesting that the frequency effect was simply a few percent larger than the AoA effect.

For the interpretation of these data, it is important to keep in mind that what is shown in Table 1 (and in the subsequent tables) are pure effects of AoA and frequency, controlled for the other variable (e.g., AoA controlled for frequency). With unselected stimulus materials, there always is a strong correlation between the effects of AoA and frequency, but this correlation is rather uninformative as the two variables are highly intercorrelated (i.e., most early acquired words have a high frequency, and most low-frequency words are acquired late). So, what Table 1 shows is that even for those stimuli that have been selected specifically to partial out the intercorrelation of AoA and frequency, there still is a near perfect correlation of the effects due to AoA and frequency in a wide range of verbal tasks. 
Table 1 : Effects of AoA and frequency in six different tasks based on the same set of English words (Gerhand \& Barry, 1998, 1999a, 1999b).

\begin{tabular}{llc}
\hline & AoA effect & frequency effect \\
& & \\
Immediate naming & $14 \mathrm{~ms}$ & $22 \mathrm{~ms}$ \\
Delayed naming & $-11 \mathrm{~ms}$ & $-1 \mathrm{~ms}$ \\
Speeded naming & $28 \mathrm{~ms}$ & $26 \mathrm{~ms}$ \\
Lexical decision (legal nonwords) & $54 \mathrm{~ms}$ & $77 \mathrm{~ms}$ \\
Lexical decision (illegal nonwords) & $24 \mathrm{~ms}$ & $33 \mathrm{~ms}$ \\
Lexical decision (pseudohomophon.) & $56 \mathrm{~ms}$ & $90 \mathrm{~ms}$ \\
\hline
\end{tabular}

The second multitask investigation was reported by Ghyselinck, Lewis, and Brysbaert (2004a). They used the same six tasks as Gerhand and Barry with Dutch stimulus words, and added two more tasks. The first was a semantic categorisation task. Participants had to decide whether a string of letters formed a noun (e.g., pan) or the first name of a person (e.g., pam; see Brysbaert, Van Wijnendaele, \& De Deyne, 2000). The second task was perceptual identification with the words presented for 33 ms. There were 96 words, equally divided in four groups: 24 early acquired (< 7 years) low-frequency (<3/million) nouns, 24 early acquired high-frequency (> 25/million) nouns, 24 late-acquired (> 10 years) low-frequency nouns, and 24 late-acquired high-frequency nouns. The data are shown in Table 2. Again, the most conspicuous finding was the strong positive correlation between the effects of AoA and frequency across tasks $(r=.97, n$ = 7). When the AoA effect was small, the frequency effect was small, and vice versa. Again, the intercept of the regression line did not differ from 0 . However, with this set of stimuli, the frequency effect was smaller than the AoA effect (in contrast with Table 1). Finally, Ghyselinck 
et al. (2004a) reported a significant AoA effect in the perceptual identification task (contrary to Gilhooly \& Logie, 1981).

Table 2 : Effects of AoA and frequency in eight different tasks based on the same set of Dutch words (Ghyselinck, Lewis, \& Brysbaert, 2004).

\begin{tabular}{lcc}
\hline & AoA effect & frequency effect \\
& & \\
& $17 \mathrm{~ms}$ & $9 \mathrm{~ms}$ \\
Immediate naming & $-5 \mathrm{~ms}$ & $14 \mathrm{~ms}$ \\
Delayed naming & $14 \mathrm{~ms}$ & $9 \mathrm{~ms}$ \\
Speeded naming & $75 \mathrm{~ms}$ & $70 \mathrm{~ms}$ \\
Lexical decision (legal nonwords) & $12 \mathrm{~ms}$ & $18 \mathrm{~ms}$ \\
Lexical decision (illegal nonwords) & $116 \mathrm{~ms}$ & $102 \mathrm{~ms}$ \\
Lexical decision (pseudohomophon.) & $52 \mathrm{~ms}$ & $35 \mathrm{~ms}$ \\
Semantic categorisation (names) & & $10 \%$ \\
\hline
\end{tabular}

The third multitask investigation was run by Chalard (2002) in French. She asked participants to name and make lexical decisions to early and late acquired words, matched for frequency, and to low- and high-frequency words matched for AoA. The data of these experiments (see the first two lines of Table 3) are well in line with the others described in Tables 1 and 2. There were rather small and equivalent effects of AoA and frequency for naming, and stronger effects for lexical decision, with the effect of AoA slightly bigger than that of frequency. A similar pattern emerged from multiple regression analyses on a larger set of 237 words. In these analyses too, the $\beta$-values of AoA and frequency were yoked ${ }^{1}$, both in naming (AoA: $\beta=.136$; Freq: $\beta=-.088$ ) and in lexical decision (AoA: $\beta=.297$; Freq: $\beta=-.266$ ).

\footnotetext{
${ }^{1} \beta$-values or standardised regression coefficients are obtained when the regression analysis is done on the $z$-values of the dependent and the independent variables. In this way, the values are not influenced
} 
A completely different pattern emerged, however, when the stimuli were pictures of objects rather than words. Whereas the frequency effect remained the same as in word naming, the AoA effect increased dramatically when participants were asked to say or to write down the names of the objects (see the last two lines of Table 3). A similar effect was observed in the multiple regression analyses, where the $\beta$-value of AoA became tenfold that of frequency. This finding (see also Bonin, Fayol, \& Chalard, 2001) is not limited to the French language, as the same pattern can be discerned in English, when one compares the data of Ellis and Morrison (1998; object naming) with those of Morrison and Ellis (2000; word naming and lexical decision). Unfortunately, these authors considerably obscured the pattern by presenting nonstandardised regression weights as $\beta$-coefficients and by using different AoA measures in both papers (AoA vs. $\log (\mathrm{AoA}))$.

Table 3 : Effects of AoA and frequency in four different tasks based on French stimuli (Chalard, 2002).

\begin{tabular}{lcl}
\hline & AoA effect & frequency effect \\
& $28 \mathrm{~ms}$ & $15 \mathrm{~ms}$ \\
Word naming & $66 \mathrm{~ms}$ & $49 \mathrm{~ms}$ \\
Lexical decision (legal nonwords) & & \\
Picture naming & $147 \mathrm{~ms}$ & $10 \mathrm{~ms}$ \\
Picture writing & $81 \mathrm{~ms}$ & $15 \mathrm{~ms}$ \\
\hline
\end{tabular}

All in all, a rather consistent and intriguing picture emerges from the multitask investigations. AoA seems to have two different effects: one that is yoked to the frequency

by differences in the variance of the independent variables (see Aron \& Aron, 2003, pp. 114-124, for a good explanation). 
effect, and one that is independent of the frequency effect (see Bates, Burani, D’Amico, \& Barca, 2001, for a similar conclusion based on a factor analysis of Italian word reading and picture naming tasks). As long as the stimuli are words and the task involves a simple response to these words, there is a nearly perfect positive correlation between the magnitude of the frequency effect and the magnitude of the AoA effect over a whole range of tasks. In addition, both effects are pretty similar in magnitude. When the range of AoA values is stretched to the maximum (say, early acquired words learned before 4 years old and late acquired words learned after 10 years) at the expense of the frequency range, the AoA effect is a few percent bigger than the frequency effect (Ghyselinck et al.; Chalard). In contrast, when the AoA range is slightly restricted to have a maximal frequency difference, the frequency effect is a few percent larger (Gerhand \& Barry). In multiple regression analyses, the $\beta$-coefficients of both variables are also very similar (Chalard).

The pattern of data changes dramatically when the stimuli are pictured objects rather than words. When these stimuli have to be named (either orally or in written form), the AoA effect no longer stays in tune with the frequency effect, but exceeds it to a large extent. In the past, the strong AoA effect in object naming has been interpreted as evidence for the idea that the AoA effect is localised in the speech output system. However, such an interpretation rests uneasily with the word processing data, where there is no clear dissociation between naming and lexical decision tasks. So, the unusually strong effect of AoA in object naming is likely to have another origin than the production of the object names themselves. Below, we will elaborate on the most likely interpretations of the frequency-related and the frequency-independent AoA effects.

\section{Interpretations of the frequency-related AoA effect}

As indicated in Tables 1-3, part of the effect ascribed to age-of-acquisition is strongly related to the frequency effect. This finding fits easily within the cumulative-frequency 
hypothesis of the AoA effect (Lewis, 1999). According to this hypothesis, processing time of a stimulus depends on the total number of times the stimulus has been encountered in the past. This total (or cumulative) number is a function not only of how often the stimulus is encountered in daily life, but also of how long the stimulus has been known (which is an inverse function of AoA; e.g., a word acquired at the age of 4 is known for 16 years to a 20 -year old, whereas a word acquired at the age of 12 is known for 8 years only).

In the cumulative frequency view, it does not make a difference whether extra encounters come from more years known or from more frequent occurrences in everyday life. Each encounter is simply added to the previous and has equal weight. This leads to the expectation that in general the frequency effect should be larger than the AoA effect, because word frequencies have a much wider range than AoAs. Frequencies typically range from less than 10 per million to more than 100 per million. This results in a ratio that can easily exceed 20:1. In contrast, few words are learned before the age of 2 or later than the age of 14 (certainly if they have to be matched on frequency). This makes a ratio of years known equal to 18:6 (3:1) for 20year olds, and equal to 78:66 (1.2:1) for 80-year olds. Looking at the multitask investigations listed in Tables 1-3, the situation is even worse, with the early acquired words usually learned around the age of 5 and the late acquired words around the age of 10. For a 20-year old this makes a ratio of 15:10 (1.5:1); for an 80-year old, the ratio decreases to 75:70 (1.1:1).

The above calculations make clear why the cumulative frequency hypothesis cannot account for the AoA effects observed in Tables 1-3. If the frequency-related AoA effects were due to cumulative frequency, they should be much smaller than the frequency effects (although the magnitudes of both effects would still correlate perfectly across tasks). In addition, we would have to expect that the AoA effect decreases as the participants grow older, a finding not obtained by Morrison, Hirsh, Chappell, and Ellis (2002) and by Lewis, Chadwick, and Ellis (2002). In these studies, the AoA effect remained as important for elderly as for youngsters. 
A model that better accounts for the two failed predictions of the cumulative frequency hypothesis, is the neural-network account, first presented by Ellis and Lambon Ralph (2000). When a neural network with distributed input and output is trained in such a way that some stimuli are entered earlier than others but continue to be presented throughout the learning phase (i.e., the early trained items are not replaced by the later items), there is an advantage for the items that were introduced early into the network. In addition, the advantage of the early acquired words survives huge differences in cumulative frequency, even though the frequency of presentations has a clear effect on network performance as well. On the basis of their simulations, Ellis and Lambon Ralph (2000) argued that the AoA effect arises as a natural property of systems in which learning takes place in a cumulative and interleaved manner, and they attributed this effect to the gradual loss of plasticity in the learning system. When the network is still young, new words can cause large weight shifts in the connections from the input units to the output units. As the network gets older, the extent of the weight shifts becomes smaller because the connection strengths are already close to one of the extremes (either 0.0 or 1.0). Therefore, the weight shifts induced by later-acquired words will never be as substantial as those induced by early-learned ones. As a consequence, the words that are learned early in training will be have a larger influence on the final structure of the network.

Because the neural-network account explains the basic findings on AoA as an emergent property of the training, in the last two or three years it has rapidly become the dominant framework to interpret AoA effects, and it would seem to be the prime candidate for the explanation of the frequency-related AoA effects in Tables 1-3 as well. It can explain (a) why the effects of AoA and frequency are highly correlated (because both are based on the same learning mechanism), (b) why there is a stronger effect of AoA than expected on the basis of cumulative frequency, and (c) why the effect of AoA does not decrease with the age of the participants.

On the other hand, the success of the neural-network account must not make us forget that there are also a few problems with it. The first one was highlighted by Zevin and Seidenberg 
(2002). They agreed with Ellis and Lambon Ralph (2000) that the acquisition order determines the entrenchment of a neural network, but emphasised much more that the emergence of an AoA effect, over and above what is expected on the basis of the cumulative frequency effect, depends on the sort of task that has to be performed. The acquisition order is particularly important when the mapping between input and output is arbitrary; that is, when no generalisation of early trained patterns to later trained patterns is possible. Otherwise, the regularities learned for the early-acquired patterns are transferred to the later-acquired patterns. Specifically with respect to visual word naming, Zevin and Seidenberg (2002) argued that the mapping from orthography to phonology in English is not arbitrary enough to give rise to an AoA effect over and above the cumulative frequency effect (because many onsets and rimes of words are consistent between early-learned and late-learned words; e.g., the rime of the late acquired SPAT is the same as that of the early acquired CAT). Given that the grapheme-phoneme mappings are even more consistent in Dutch and French than in English, for these languages one would certainly expect an AoA effect in word naming that is significantly smaller than the frequency effect (given the difference in the ranges of frequency and years known). This is not what has been obtained in Tables 1-3: The AoA effect is equally strong relative to the frequency effect in word naming as in any other task with visual word stimuli.

The second problem with the neural-network account is that it is based on distributed representations (i.e., representations that fully overlap one another and that have no nodes dedicated to individual words). In the simulations reported by Ellis and Lambon-Ralph (2000), the network typically consisted of a layer of 100 input units connected -via hidden units- to a layer of 100 output units. Each input pattern consisted of a random sequence of 50 zeros and 50 ones distributed over the entire input layer. The output also consisted of a random sequence of 50 zeros and 50 ones distributed over the output layer. Matched input and output patterns became associated with one another by a learning process that changed the connection weights. In other words, Ellis and Lambon-Ralph's neural networks contains no organisation at all within the 
layers; all the knowledge is stored in the connections between. In addition, because the inputs and outputs are distributed across the entire layer, each new stimulus overwrites the previous stimulus, so that the model cannot keep track of two stimuli simultaneously. All it can do, is to convert one pattern into another. Inherently, there is nothing wrong with such an organisation, except that at present no-one knows how to integrate such a three-layer network within a larger model of visual word processing. How is a string of printed letters (some of which are the same) converted into a random input pattern, and how is a random output pattern transferred into a meaningful signal that can be used, for instance, in sentence reading (which requires several words to be kept active in working memory)? Bowers (2002) recently made the case that no existing model of visual word recognition incorporates this radical type of distributed representations, not even the parallel-distributed-processing models (which are all based on localist representations for letters and sounds, and sometimes even for words). However, as soon as localist representations are used, the simulations of Zevin and Seidenberg (2002) show how difficult it is to find an AoA effect. Even the inconsistencies in the letter-sound mappings in English are not large enough to induce a genuine AoA effect in a network with localist representations for letters and sounds. So, although the neural-network account provides us with a tempting explanation of the AoA effect, we have to admit that at present nobody knows how to reconcile the requirement of distributed random representations with the necessity to pass on verbal information through a series of processing stages going from printed input to discourse interpretation. In the end, this deadlock may imply that we will have to give up the neuralnetwork account and return to the idea that the effect of AoA does not reside in the connections between representations but in the representations themselves (e.g., in the activation levels of the units).

Whatever the exact interpretation of the frequency-related AoA effect, the cumulative frequency hypothesis and the neural-network account have provided us with the tools to believe that the frequency-related AoA effect is likely to be due to the same learning process as the one 
underlying the frequency effect. For this reason, wherever there is a frequency effect, we are bound to find an AoA effect (implying that further attempts to find a frequency effect without an accompanying AoA effect are futile). In addition, current empirical evidence makes it clear that the frequency-related AoA effect is larger than expected on the basis of the cumulative frequency. On average, the AoA effect is of the same size as the frequency effect, despite the fact that the range of years-known is much smaller (sometimes to a factor of 10) than the range of frequencies-of-occurrence. If one correspondence is to be noted between the frequency-related AoA effect and the frequency effect, then it is that their standardised regression coefficients (the $\beta s)$ seem to be the same, rather than their raw-score regression weights.

\section{Interpretations of the frequency-independent AoA effect}

As shown in Table 3, the magnitude of the AoA effect deviates from the frequency effect in the picture naming task (both with oral and written responses). One difference in the processing requirements of object naming on the one hand, and word naming and lexical decision on the other hand, is that the latter tasks can be performed without semantic mediation, whereas the former cannot. There are no brain damaged patients reported in the literature, who could name objects without having at least some crude idea about what they meant (e.g., Hodges \& Greene, 1998). In contrast, many patients have been reported who could name words (even those with irregular spellings, such as yacht) and decide between words and non-words without having a clue about their meaning (e.g., Gerhand, 2001).

So, the activation of semantic information would seem to be a prime candidate for the explanation of the frequency-independent AoA effect. This semantic hypothesis has been disfavoured for a long time, because Morrison et al. (1992) failed to find an effect of AoA in a man-made vs. naturally-occurring (e.g., anchor vs. apple) decision task, whereas they found a huge AoA effect in a naming task with the same stimuli. Brysbaert et al. (2000) suggested that 
one reason for this null-effect may have been that Morrison et al. reported aggregate RTs over the two semantic classes. This may have been suboptimal, because binary decisions are often translated into a yes/no decision with different criteria for the no-responses than for the yesresponses. Another possibility for the null-result may is that decisions in a binary semantic classification task can be based on partial information of the stimulus (e.g., one need not identify a picture as a leopard to decide that it belongs to the class of the naturally-occurring things; a simple identification as an animal or a catlike would do; if the stimuli are not well chosen, a perceptual distinction between round and angular might even suffice, as man-made objects usually have more straight lines and sharp corners than living organisms).

In recent years, the semantic hypothesis has regained impetus, partly due to Brysbaert et al. (2000; also see van Loon-Vervoorn, 1989; and Bates et al., 2001), partly due to the neuralnetwork account which predicts AoA effects for arbitrary mappings. As the relations between orthography and semantics are arbitrary (very few words that are orthographically similar also mean the same), the neural-network account predicts AoA effects over and above cumulative frequency effects for semantic tasks and verbal input. Other models also predict an effect of AoA on the activation of semantic information, but these models explain the effect more in terms of the organisation of the semantic system rather than the weights of the connections to and from the system. For example, Steyvers and Tenenbaum (submitted) presented a mathematical model of a growing semantic network. The network consists of interconnected nodes that represent concepts (i.e., localist representations), and it develops according to a principle Steyvers and Tenenbaum previously observed in many different types of networks (going from the internet to the semantic memory). Basically, the principle implies that new concepts are added to the network by connecting them to existing nodes (concepts) as a function of the number of connections each node already has. This preferential-attachment principle makes the prediction that early acquired nodes have a more central position in the network because on average they have more connections than later-acquired nodes (i.e., they are more likely to be central hubs 
within the network). We ourselves (Brysbaert, Ghyselinck, \& Storms, 2002) have conjectured the idea that early acquired concepts may be more defining instances of categories than later acquired concepts (i.e., the concept "fruit" in Western cultures is defined very much in terms of apples, oranges and bananas, because these are the types of fruit infants first are confronted with; see Storms, De Boeck, \& Ruts, 2000, for evidence that natural language categories are more likely to be defined on a limited number of exemplars than on prototypes).

Research in the last few years has shown that Morrison et al. (1992) were indeed too hasty to reject an AoA effect in semantic tasks. Several studies have now established that the meaning of early acquired concepts is activated more rapidly than the meaning of late acquired concepts. However, as indicated in the previous section, this is not the main question to ask. Wherever we find a frequency effect, we are bound to find an AoA effect of the same size. The really important question is whether the AoA effect is larger than is predicted on the basis of the frequency effect; that is, whether the semantic system can be considered as the origin of the frequency-independent AoA effect. To look at this possibility, we listed all the experiments that simultaneously addressed the main effects of AoA and frequency in meaning-based tasks (see Table 4). 
Table 4 : Effects of AoA and frequency in meaning-based tasks as a function of the input format (verbal vs. pictorial)

Source

Verbal stimulus materials

Brysbaert et al. (2000)

Ghyselinck (2000, Ch. 6, Exp. 1)

Ghyselinck (2000, Ch. 6, Exp. 2)

Ghyselink et al. (2004a)

Brysbaert et al. (2000)

Catling \& Johnston (in press)

Pictorial stimulus materials

Chalard (2000)

Chalard (2000)

Meschyan \& Hernandez (2002)

Moore et al. (2004, Exp. 1) task

common noun/first name decision

man-made/naturally occurring decision

semantic category membership decision

common noun/first name decision

word associate generation

category exemplar generation
AoA effect

Frequency effect picture naming

picture writing

picture naming

object/non-object decision

$\begin{array}{rr}63 \mathrm{~ms} & 47 \mathrm{~ms} \\ 49 \mathrm{~ms} & 92 \mathrm{~ms} \\ 24 \mathrm{~ms} & 21 \mathrm{~ms} \\ 52 \mathrm{~ms} & 35 \mathrm{~ms} \\ 279 \mathrm{~ms} & -218 \mathrm{~ms} \\ 167 \mathrm{~ms} & 6 \mathrm{~ms}\end{array}$

$147 \mathrm{~ms} \quad 10 \mathrm{~ms}$

$81 \mathrm{~ms} \quad 15 \mathrm{~ms}$

$116 \mathrm{~ms} \quad 31 \mathrm{~ms}$

As is clear from Table 4, the evidence for a pure semantic origin of the frequencyindependent effect is not very compelling. In particular, three tasks with verbal input failed to provide AoA effects that went beyond the frequency effect. Brysbaert et al. (2000) asked participants to decide whether words referred to common nouns (e.g., bed) or to first names (ben), an experiment replicated by Ghyselinck et al. (2004a; see also Table 2). Despite the purely arbitrary relation between the letter sequence and the decision criterion, the AoA effect was not larger than expected on the basis of the frequency effect (Table 2). Similarly, Ghyselinck (2002, Chapter 6, Experiment 1) reported equivalent AoA and frequency effects in a carefully controlled man-made yes/no classification task, in which all the critical stimuli were names of man-made objects, to avoid the averaging of yes and no responses. Ghyselinck (2002, Chapter 6, Experiment 2) subsequently used a semantic verification task and obtained a frequency-related AoA effect as well. In this task, two words appeared simultaneously on the screen: a category label in capital letters and a target word in lower-case letters. Participants had to indicate whether the target word in lower-case letters was a valid member of the category by pressing one of two 
response buttons (e.g., FLOWER - snowdrop). Again, equivalent effects of AoA and frequency were found for the yes-trials.

Two other studies with verbal input, however, did reveal a dissociation between AoA and frequency, similar to the one found in picture naming. It were both studies in which a different word had to be generated than the one that was presented. In the first study, participants were asked to generate a word associate upon seeing a target word (e.g., "Which word comes first to your mind when you see the word dog?”). The time needed to generate the associate was substantially (over $200 \mathrm{~ms}$ ) shorter for early acquired words than for late acquired words (Brysbaert et al., 2000). No equivalent effect was observed for word frequency. As a matter of fact, a reversed frequency effect was found, because participants were faster to generate associates to low-frequency targets than to high-frequency targets when the stimuli were controlled for AoA. The second task that yielded an AoA effect without a corresponding frequency effect (Catling \& Johnston, in press), was a task in which participants got a category word (e.g., VEGETABLE) together with the first letter of an item belonging to that category (e.g., C). Participants were asked to generate the item starting with this letter (i.e., Carrot).

The evidence with pictorial stimuli is nearly exclusively based on picture naming (i.e., Chalard's studies listed in Table 3, and a study in English by Meschyan \& Hernandez). The only other study that orthogonally manipulated frequency and AoA, was reported by Moore, SmithSpark, and Valentine (in press). In this study, participants saw a picture presented for $200 \mathrm{~ms}$ between a forward and a backward mask, and had to decide wether the picture depicted a real object or a made-up nonobject. Participants were faster to early acquired objects than to lateacquired objects, but slower to objects with a high-frequency name than to objects with a lowfrequency name. Unfortunately, this finding is not corroborated by the correlational studies that looked at the same effects in binary semantic classification tasks. These studies failed to provide evidence for an AoA effect that was considerably stronger than the frequency effect (Morrison et al., 1992; Vitkovitch \& Tyrrell, 1995). Moore et al.’s conclusions are also out of line with the 
findings reported by Lewis and colleagues in two different studies on face perception (Lewis, 1999; Lewis et al., 2002). They asked participants to classify faces as belonging to characters of one of two different soap operas, and found very similar regression coefficients for the time since the first appearance on the show (a measure of AoA) and the frequency of occurrence per episode. One reason for the deviating results of Moore et al. might be the tachistoscopic presentation of the stimuli. In a series of unpublished studies, De Deyne (Ghent University) also found some evidence for larger AoA effects in semantic classification experiments with pictorial stimuli, when the stimuli were displayed tachistoscopically.

All the other studies that reported evidence for an AoA effect in meaning-based tasks, did not examine the magnitude of the frequency effect for the task at hand (see also Holmes \& Ellis in this issue). Moore and Valentine (1999) reported a $40 \mathrm{~ms}$ AoA effect in a face familiarity decision task (“Are these faces of famous people? yes/no). Chalard (2002, Experiment 3), using pictures as stimuli, reported a $23 \mathrm{~ms}$ AoA effect in an object/non-object decision task. Johnston and Barry (2002), also using pictorial stimuli, reported that early acquired objects were categorised some 50-60 ms faster than late acquired objects in an "inside/outside the house” classification task and in a "smaller or larger than a loaf” classification task. Ghyselinck, Custers, and Brysbaert (2004b) asked participants to say "living” when words were presented in uppercase, and "non-living” when words were presented in lowercase letters. Half of the words in each letter condition effectively referred to living things (CHICKEN, vulture), half to nonliving things (CORD, dome). Ghyselinck et al. obtained a congruency effect, because participants found it more difficult to say "living" to the uppercase stimulus CORD than to the uppercase stimulus CHICKEN. Similarly, they found it more difficult to say "non-living" to the lowercase stimulus vulture than to the lowercase stimulus dome. This congruency effect was significantly larger for early acquired words (CHICKEN/chicken: $50 \mathrm{~ms}$ ) than for late acquired words (VULTURE/vulture: $25 \mathrm{~ms}$ ). Unfortunately, none of these authors tested the effects of word (or concept) frequency in their tasks. 
All in all, although the activation of semantic information may be involved in the frequency-independent AoA effect, the evidence presented in Table 4 is not very convincing. Quite some AoA effects reported in meaning-based tasks are (or could turn out to be) frequencyrelated AoA effects. This is especially true for the studies that used verbal input and/or a binary decision task. Only in those studies that required a specific word to be generated on the basis of a semantic analysis of the input, seemed to provoke the frequency-independent AoA effect ${ }^{2}$. So, it may be informative to try to find out what is specific to this particular situation. Two ideas come to mind. First, correct performance in naming objects and generating word associates depends on the full activation of one single semantic concept, rather than on the partial activation of a broad semantic category. Second, naming objects and generating associates involves a competition among several candidates before a response can be emitted.

It is now well-accepted that stimuli need not be fully identified to make binary decisions. This can easily be seen in lexical decisions with different types of non-words. For instance, in Ghyselinck et al. (2004a), decisions to words took on average 815 ms when the non-words were pseudohomophones (brane), and only $512 \mathrm{~ms}$ when the non-words were illegal letter strings (brnkf). Although some of this difference could be explained by assuming that in the former experiment participants based their decision primarily on the word stimuli, whereas in the latter experiment they based their decision more on the non-word stimuli, this is unlikely to be the whole story, given the effects of frequency and AoA in the experiment with illegal non-words. Rather, what seems to happen is that in both experiments evidence for word-like stimuli was accumulated and used, but that the threshold value to make a word decision was lower when the non-words were illegal letter strings than when they were pseudohomophones (Gibbs \& Van Orden, 1998; Grainger \& Jacobs, 1996). Similarly, what is likely to happen when participants are making a decision between, for instance, man-made vs. naturally-occurring stimuli is that all the evidence for one or the other category is accumulated until a threshold criterion is exceeded,

\footnotetext{
${ }^{2}$ Notice that this exactly may be what the tachistoscopic presentation of Moore et al. induced: A need to
} 
with the value of the threshold depending on the overlap of the two categories. Critically, however, the accumulation of evidence depends not only on the specific stimulus presented, but also on other stimuli that are temporarily co-activated in the process of stimulus recognition. For instance, Rodd (2003) observed that participants found it more difficult to reject the stimulus word leotard as an animal than to reject a matched control word that did not orthographically resemble the name of an animal, presumably because the meaning of the orthographically related word (leopard) became temporarily co-activated in the process of word recognition. Similarly, from the semantic priming literature (e.g., Lucas, 2000) we know that a stimulus word such as leopard (or the picture of a leopard) is likely to automatically co-activate the words (concepts) of tiger, cat, and lion, maybe making an "animal” decision for this stimulus easier than for worm (which does not have these obvious animal associates).

So, one reason why the AoA effect is stronger in object naming and in associate generation than in semantic classification may be that in the former tasks a unique concept must be selected. This is likely to involve a stronger competition at the conceptual level than a binary decision which can be based on converging evidence from different concepts, both early and late acquired (e.g., the co-activation of the concepts mouse and rat upon seeing the picture of a guinea pig helps to make an "animal” decision, but hinders the singling out of the specifically presented stimulus). Assuming that the early acquired concepts are stronger competitors than later acquired concepts, this might explain the frequency-independent AoA effect in object naming and associate generation. Needless to say, the assumption would be more convincing if we could find independent empirical evidence for it.

Alternatively, because the object naming and associate generation involve the production of a verbal response, it might be hypothesised that the competition between early and late acquired words does not happen at the conceptual, meaning level, but at the level where the correct output is chosen. Models of word production (e.g., Dell, Schwartz, Martin, Saffran, \& 
Gagnon, 1997; Levelt, Roelofs, \& Meyers, 1999) distinguish three processing levels from message to word production: a conceptual level, a lemma level (which contains lexical entries pointing to the meaning and the syntactic information of words), and a phonological word-form (lexeme) level. One obvious candidate for the competition, besides the conceptual level, would seem to be the lemma level, because many speech production theories assume that, during the lemma selection process, lemmas which partially match the conceptual structure are activated to some extent, and compete for selection. This competition can be observed in the picture-word interference task. When participants have to produce the name of a picture, they are particularly slowed down when slightly before or simultaneously with the onset of the picture they hear a semantically related word (e.g., they have to name the picture of a cat while hearing the word “dog”; Damian \& Martin, 1999). The idea is that the presentation of a semantically related intruder increases the competition at the lemma level, so that it becomes harder to overcome the interference. Even without the presentation of a verbal intruder, it is likely that many pictures activate more than one lemma, as can be concluded from the fact that the degree of name agreement about a particular picture is a significant predictor of naming latencies (e.g., Chalard, 2002). As a matter of fact, it is standard practice in picture naming tasks first to go through the pictures and to make sure that all participants agree with the proposed names. Response competition is also likely to occur in word associate generation, where different possible candidates become partly activated before one is eventually produced.

Attentive readers may have noticed that the second explanation (competition at the lemma level) comes quite close to the phonological output hypothesis that was popular at the onset of the AoA research in the 1990s (Morrison \& Ellis, 1995; Gerhand \& Barry, 1998). However, there are two important differences. First, the phonological output hypothesis was strongly influenced by Brown and Watson’s (1987) idea that the phonological representations of the early acquired words were stored in a complete form in the speech output system, whereas the representations of later learned words were stored in fragments and had to be assembled 
before the word could be produced. In contrast, our proposal is that the AoA effect arises out of a competition between different possible outputs. This is in line with the finding that the AoA effect is not larger than expected on the basis of frequency in word naming (where the phonological word form has to be produced but where there is no response competition). The second difference is that the locus of the AoA effect in the phonological output hypothesis was firmly placed at the level of the phonological word forms (lexemes), whereas we see it more likely at the interface between semantics and word production (the lemma level) and/or at the conceptual level.

Attentive readers may also have noticed that our proposals disagree with Levelt et al. (1999, pp. 18-19), who gave reasons why they thought the frequency effect (and the AoA effect) had to be situated at the lexeme level. They rejected the conceptual level as the origin, because Jescheniak and Levelt (1994) had failed to find a frequency effect in a picture recognition task. Participants were given the name of a stimulus (e.g., "boat") and subsequently shown a picture; they had to indicate whether the name and the picture agreed or not. There was a frequency effect neither in the "yes" responses nor in the "no" responses, leading the authors to conclude that frequency was not involved in the activation of semantic information. However, a problem with Jescheniak and Levelt's task is that the presentation of the stimulus name before the picture is likely to have induced a massive priming effect, which is known to reduce and sometimes to eliminate the frequency effect, because priming is more substantial for low-frequency targets than for high-frequency targets (Becker, 1979). As reviewed above, other carefully controlled semantic categorisation tasks have resulted in frequency and AoA effects, even with pictorial stimuli. Levelt et al.’s (1999) main argument for attributing the word frequency effect to word form access rather than to lemma selection, stemmed from an experiment, also described by Jescheniak and Levelt (1994), in which participants produced polysemous words ${ }^{3}$. Proficient

\footnotetext{
${ }^{3}$ Interestingly, Levelt and colleagues never refer to their stimuli as polysemous words. They always refer to them as homophones and give examples of English heterographic homophones when they describe their manipulation (e.g., they say the words were of the type wee - we, moor - more, whereas in reality
} 
Dutch-English bilingual participants were asked to translate English words into their mother tongue. The translations of some English words were low-frequency Dutch words which had another, higher-frequency meaning (e.g., the Dutch translation of the English word layer is laag; however, the Dutch word laag is more often used as an adjective, meaning low). Translation times for these polysemous stimuli were compared to those of matched low-frequency monosemous words (e.g., wage - loon), and matched high-frequency monosemous words (e.g., afraid - bang). Translation times for the low-frequency polysemous words [layer-laag] turned out to be as fast as those for the high-frequency control words [afraid-bang], and faster than those for the low-frequency control primes [wage-loon]. Because the different meanings of words only share their lexeme and not their lemmas, Jescheniak and Levelt (1994) and Levelt et al. (1999) concluded that the frequency effect had to be situated at the lexeme level. However, as indicated above, whereas this explanation would seem to work for the frequency effect and the frequency-related AoA effect, it does not offer an explanation for the frequency-independent AoA effect, because this effect is observed in picture naming only and not in word naming, even though the lexeme is needed for good performance in both tasks.

All in all, in our view the most likely explanation of the frequency-unrelated AoA effect has to do with a competition process at the semantic (conceptual) level and/or at the interface between the conceptual level and the verbal output system (the lemma level). For some reason, early acquired words/concepts are stronger competitors than late acquired words/concepts, so that they more easily win the competition for their own recognition, and cause more trouble for the recognition of competitors.

their stimuli were of the type bank -which can be translated in two different ways in Dutch, depending on whether it refers to a river or to money). 


\section{Conclusion}

On the basis of a close examination of multitask investigations that were published in English, Dutch, and French, we have discerned a pattern that had escaped us before (and seems to have escaped many other researchers in the area). For most tasks, the AoA effect is well yoked to the frequency effect (or vice versa), even though the stimuli were selected so that the AoA and the frequency of the words were orthogonal variables. Further intriguing is that over a whole range of tasks, the AoA effect on average is of the same size as the frequency effect (resulting in equivalent $\beta$-coefficients), despite the fact that the range of AoA values (or years-known) is much smaller than the range of frequency values. This seems to rule out the most straightforward account of why AoA and frequency are two sides of the same coin, namely that they are both measures of the cumulative frequency with which a stimulus has been encountered. This means that other, more sophisticated learning algorithms will have to be found. One very promising candidate is the training that occurs in a neural network with distributed representations. The challenge for this account is not so much that it fails to explain the existing data (except maybe for the AoA effect in word naming), but that it is not clear how such a network with radically distributed representations can be integrated within a full-fledged model of word recognition and use, which unavoidably has to consist of different processing stages, with information flowing from one stage to the next (and sometimes coming back).

In addition to the frequency-related AoA effect, there is a frequency-independent effect in tasks that require a specific word to be generated on the basis of a semantic analysis of the input. We venture that the most likely origin of this effect is a competition between different representations at the conceptual and/or lemma level. This is in line with the semantic hypothesis of the AoA effect we have defended in the past (Brysbaert et al., 2000; Brysbaert et al., 2002; Ghyselinck et al.,2004b). It is also to some extent in line with a recent brain imaging study reported by Fiebach, Friederici, Müller, von Cramon, and Hernandez (2003). This study showed 
that differences in word frequency and AoA modulated brain activity in overlapping regions. However, AoA additionally modulated activity in brain areas not affected by word frequency: Early acquired words specifically activated the precuneus, a brain area associated both with episodic and semantic memory, which the authors attributed to the fact that understanding the meaning of words at a young age is more closely linked to subjective experiences than it is later in life. In addition, visually presented early acquired words activated the auditory cortex, which according to the authors may be due to the fact that until 5 or 6 years of age language is learned exclusively through the auditory modality. Although the Fiebach et al. study confirms the distinction drawn here between frequency-related and frequency-independent AoA effects, it also points to the fact that the semantic representations of early acquired words may be different from those of late acquired words (i.e., much more experience-based), a point previously made by van Loon-Vervoorn (1989) as well. This may be another factor to take into account in future research. 


\section{References}

Aron, A., \& Aron, E.N. (2003). Statistics for Psychology (3rd ed.). Upper Saddle River, NJ: Prentice Hall.

Bates, E., Burani, C., D’Amico, S., \& Barca, L. (2001). Word reading and picture naming in Italian. Memory \& Cognition, 29, 986-999.

Becker, C.A. (1979). Semantic context and word frequency effects in visual word recognition. Journal of Experimental Psychology: Human Perception and Performance, 5, 252-259.

Bonin, P., Fayol, M., \& Chalard, M (2001). Age of acquisition and word frequency in written picture naming. Quarterly Journal of Experimental Psychology, 54A, 569-489.

Bowers, J. S. (2002). Challenging the widespread assumption that connectionism and distributed representations go hand-in-hand. Cognitive Psychology, 45, 413-445.

Brown, G. D. A, \& Watson, F. L. (1987). First in, first out. Word learning age and spoken word frequency as predictors of word familiarity and word naming latency. Memory and Cognition, 15, 208-216.

Brysbaert, M. (1996). Word frequency affects naming latency in Dutch with age of acquisition controlled. European Journal of Cognitive Psychology, 8, 185-193.

Brysbaert, M., Ghyselinck, M., \& Storms, G. (2002). Effects of age of acquisition in semantic categorisation tasks. Joint Meeting of EPS and BVP/SBP, Leuven, Belgium.

Brysbaert, M., Van Wijnendaele, I, \& De Deyne, S. (2000). Age-of-acquisition of words is a significant variable in semantic tasks. Acta Psychologica, 104, 215-226.

Chalard, M. (2002). Effets de l'age d'acquisition et de la fréquence objective en production verbale orale et écrite de mots isolés. Unpublished $\mathrm{PhD}$ Thesis, Université Blaise Pascal, Clermont-Ferrand.

Dell, G.S., Schwartz, M.F., Martin, N., Saffran, E.M., \& Gagnon, D.A. (1997). Lexical access in aphasic and nonaphasic speakers. Psychological Review, 104, 801-838.

Ellis, A. W., \& Morrison, C. M. (1998). Real age-of-acquisition effects in lexical retrieval. Journal of Experimental Psychology: Learning, Memory, and Cognition, 24, 515-523.

Ellis, A. W., \& Lambon Ralph, M. A. (2000). Age of acquisition effects in adult lexical processing reflect loss of plasticity in maturing systems: Insights from connectionist networks. Journal of Experimental Psychology: Learning, Memory and Cognition, 26, 1103-1123

Fiebach, C. J., Friederici, A. D., Müller, K., von Cramon, D. Y., \& Hernandez, A. E. (2003). Distinct brain representations for early and late learned words. NeuroImage, 19, 16271637.

Gerhand, S. (2001). Routes to reading: A report of a non-semantic reader with equivalent performance on regular and exception words. Neuropsychologia, 39, 1473-1484.

Gerhand, S., \& Barry, C. (1998). Word frequency effects in oral reading are not merely age-ofacquisition effects in disguise. Journal of Experimental Psychology: Learning, Memory, and Cognition, 24, 267-283. 
Gerhand, S., \& Barry, C. (1999a). Age of acquisition, word frequency, and the role of phonology in the lexical decision task. Memory \& Cognition, 27, 592-602.

Gerhand, S., \& Barry, C. (1999b). Age-of-acquisition and frequency effects in speeded word naming. Cognition, 73, 27-36.

Gibbs, P., \& Van Orden, G. C. (1998). Pathway selection's utility for control of word recognition. Journal of Experimental Psychology: Human Perception and Perfomance, 24, 1162-1187.

Gilhooly, K. J., \& Logie, R. H. (1981). Word age-of-acquisition and visual recognition thresholds. Current Psychological Research, 1, 215-225.

Grainger, J., \& Jacobs, A.M. (1996). Orthographic processing in visual word recognition: A multiple read-out model. Psychological Review, 103, 518-565.

Gyselinck, M. (2002). Age of acquisition as an organizing variable of the semantic system. Unpublished PhD Thesis, Ghent University.

Ghyselinck, M., Custers, R., \& Brysbaert, M. (2004). The effect of age of acquisition in visual word processing: Further evidence for the semantic hypothesis. Journal of Experimental Psychology: Learning, Memory, and Cognition.

Ghyselinck, M., Lewis, M.B., \& Brysbaert, M. (2004). Age of acquisition and the cumulativefrequency hypothesis: A review of the literature and a new multi-task investigation. Acta Psychologica, 115, 43-67.

Hodges, J.R., \& Greene, J.D.W. (1998). Knowing about people and naming them: Can Alzheimer's disease patients do one without the other? Quarterly Journal of Experimental Psychology, 51A, 121-134.

Holmes, S.J., \& Ellis, A.W. (2004). Age of acquisition and typicality effects in picture naming, object decision and category verification. Visual Cognition.

Jescheniak, J.D., \& Levelt, W.J.M. (1994). Word-frequency effects in speech production: Retrieval of syntactic information and of phonological form. Journal of Experimental Psychology: Learning, Memory, and Cognition, 20, 824-843.

Johnston, R. A., \& Barry, C. (2002). Age-of-acquisition effects on two picture categorization tasks. Poster presented at the 43rd Annual Meeting of the Psychonomic Society, November 21-24, 2002, Kansas City, Missouri.

Levelt, W.J.M., Roelofs, A., \& Meyer, A.S. (1999). A theory of lexical access in speech production. Behavioral and Brain Sciences, 22, 1-76.

Lewis, M. B. (1999). Age of acquisition in face categorisation: Is there an instance-based account? Cognition, 71, 23-39.

Lewis, M. B., Chadwick, A. J., \& Ellis, H. D. (2002). Exploring a neural-network account of age-of-acquisition effects using repetition priming of faces. Memory \& Cognition, 30, 1228-1237.

Lucas, M. (2000). Semantic priming without association: A meta-analytic review. Psychonomic Bulletin \& Review, 7, 618-630. 
Meschyan, G., \& Hernandez, H. (2002). Age of acquisition and word frequency: Determinants of object-naming speed and accuracy. Memory \& Cognition, 30, 262-269.

Moore, V., Smith-Spark, J.H., \& Valentine, T. (in press). The effects of age of acquisition on object recognition. European Journal of Cognitive Psychology.

Moore, V., \& Valentine, T. (1999). The effects of age of acquisition in processing famous faces and names: Exploring the locus and proposing a mechanism. In Proceedings of the Twenty-First Annual Meeting of the Cognitive Science Society (pp. 416-421). Mahwah, NJ: Erlbaum.

Morrison, C. M., Ellis, A. W., \& Quinlan, P. T. (1992). Age of acquisition, not word frequency, affects object naming, not object recognition. Memory \& Cognition, 20, 705-714.

Morrison, C. M., \& Ellis, A. W. (1995). Roles of word frequency and age of acquisition in word naming and lexical decision. Journal of Experimental Psychology: Learning, Memory, and Cognition, 21, 116-133.

Morrison, C. M., \& Ellis, A. W. (2000). Real age of acquisition effects in word naming and lexical decision. British Journal of Psychology, 91, 167-180.

Morrison, C. M., Hirsh, K. W., Chappell, T., \& Ellis, A. W. (2002). Age and age of acquisition: An evaluation of the cumulative frequency hypothesis. European Journal of Cognitive Psychology, 14, 435-459.

Rodd, J.M. (2003). Where do leotards get there spots? Semantic activation of lexical neighbours in visual word recognition. EPS Meeting, Reading, UK.

Steyvers, M, \& Tenenbaum, J. (submitted). The large-scale structure of semantic networks: Statistical analyses and a model of semantic growth. see http://wwwpsych.stanford.edu/ msteyver/papers/smallworlds.pdf

Storms, G., De Boeck, P., \& Ruts, W. (2000). Prototype and exemplar-based information in natural language categories. Journal of Memory and Language, 42, 51-73.

van Loon-Vervoorn, W. A. (1989). Eigenschappen van basiswoorden. Lisse: Swets and Zeitlinger.

Vitkovitch, M., \& Tyrrell, L. (1995). Sources of disagreement in object naming. Quarterly Journal of Experimental Psychology, 48A, 822-848.

Zevin, J. D., \& Seidenberg, M. S. (2002). Age of acquisition effects in word reading and other tasks. Journal of Memory and Language, 47, 1-29. 